\title{
Magnetization Processes in Dilute Metamagnets with Ferro- and Antiferromagnetic Exchange Interactions
}

\author{
R. PikuŁa And J. Kirkiewicz
}

Maritime University, Department of Physics

Wały Chrobrego 1/2, 70-500 Szczecin, Poland

(Received February 27, 2006; in final form June 23, 2006)

\begin{abstract}
Using the Green function method there has been presented the mathematical description of dilute two sub-lattice metamagnets with ferro- and antiferromagnetic exchange interactions. The Green function averaging procedure is based on the Cumulant Expansion Technique and includes any localized Green function irrespectively of the decoupling algorithm making the chain system of equations self-consistent. The derived form of the averaged Green function is the base for the analysis of magnetization processes and phase transitions occurring in the system under the influence of external magnetic field. It has been found in particular that the tricritical point and the second-order phase transition vanish when concentrations of magnetic atoms coupled with different sub-lattice points become different.
\end{abstract}

PACS numbers: 05.70.Jk, 64.60.Kw, 75.10.-b, 75.30.Kz

\section{Introduction}

Among the papers, which discuss properties of disordered magnets, the ones describing dilute magnets with structural disorder are in the greatest number. The papers give descriptions of the systems consisting of magnetic atoms (of one or many types) and non-magnetic atoms or only magnetic atoms of different types randomly coupled with the crystal lattice points. According to the electronic structure of the components the magnets are divided into metallic alloys and dilute magnets with stable magnetic moments located at the lattice sites. The latter group of magnets is mostly described in reference to binary systems including, besides non-magnetic atoms, also magnetic atoms with one kind of exchange interactions. Theoretical description of magnets of this type is usually made on the basis of two models. One model takes into account only fluctuations in the number of magnetic atoms coupled with lattice points (site model) but in the other the presence of non-magnetic atoms or vacancies is replaced with fluctuations of 
exchange interactions (bond model). In both the approaches the properties of dilute magnets with two types of magnetic components and two kinds of exchange interactions are also analysed [1-8]. However, the disorder which is most often discussed relates usually to simple systems containing non-interacting magnetic atoms of different types randomly distributed over all lattice points in the whole system. The structural disorder of this type is the only one possible in the case when the system consists of magnetic atoms of the same type and non-magnetic atoms that relates to the model of dilute ferro- or antiferromagnet. In dilute magnets containing at least two types of magnetic components it is possible to realize a substantial case of partial structural disorder which connects with the presence of spatial anisotropy of exchange interactions and sub-lattice structure of a system. In this case the magnetic components may be distributed in two possible ways depending whether the lattice points on different sub-lattices are occupied with the same or different probability. A wide class of ferrimagnetic materials of both the types is available. A selective distribution of magnetic atoms occurs for some dilute ferrimagnets with the perovskite structure and mixed ferromagnetic intraand antiferromagnetic inter-sub-lattice exchange interactions. Such magnetic compounds as $\mathrm{LaMn}_{1-c} \mathrm{Ga}_{c} \mathrm{O}_{3}, \mathrm{La}_{1-c} \mathrm{X}_{c} \mathrm{MnO}_{3}$, with $\mathrm{X}=\mathrm{Ca}$, Ba, Sr or $\mathrm{La}_{1-c} \mathrm{Sr}_{c} \mathrm{CoO}_{3}$ show an asymmetrical sub-lattice structure due to the non-magnetic atoms of a concentration $c$ randomly occupying, in a given range of $c$, the lattice points within one of two sub-lattices only. Other impurity ions such as, for instance, $\mathrm{Ni}^{3+}$ and $\mathrm{Co}^{3+}$ in ferrimagnets $\mathrm{LaMn}_{1-c} \mathrm{Ni}_{c} \mathrm{O}_{3}$ or $\mathrm{LaMn}_{1-c} \mathrm{Co}_{c} \mathrm{O}_{3}$ do not have this property and they are distributed over all lattice points in completely unordered fashion [9]. The experimental investigations usually show the effect of content of impurity ions on magnetization and the critical temperature of the compounds in the case of zero external magnetic field.

The aim of this paper is a presentation of the magnetization processes and phase transitions occurring under the influence of the external magnetic field in dilute magnets containing two kinds of magnetic atoms and non-magnetic components. The mathematical description relates to the two-sub-lattice system of the site type with ferromagnetic exchange interactions within each of the sublattice and antiferromagnetic exchange interactions between magnetic atoms on different sub-lattices. There has been presented the general computational model using Green function and its averaging procedure over all possible distributions of magnetic atoms in the system. The averaging procedure includes any localized Green function whose analytical form depends on the method of decoupling of the chain system of equations as well as on the equation's order at which the decoupling is done. By the use of the Cumulant Expansion Technique it was possible to present the averaged Green function in the infinite sequence of all correlations of structural fluctuations resulting from a random distribution of magnetic atoms in the system. This paper presents the simple model of summing of the elements of the obtained expansion considering its most substantial contributions coming 
from the square correlations of structural fluctuations which act the predominant role in the whole range of the concentrations of magnetic atoms. The derived analytical form of the averaged Green function makes possible the analysis of the magnetization processes and critical phenomena in the external magnetic field for various temperatures and concentrations of magnetic components.

\section{Hamiltonian}

Here we consider a crystal lattice retaining translational symmetry and divided into two geometrically equivalent sub-lattices $\alpha$ and $\beta$. Among all $N$ lattice points, $m$ points are randomly coupled with magnetic atoms whereas $N-m$ points are coupled with non-magnetic atoms. It is assumed that there are ferro- and antiferromagnetic exchange interactions between magnetic atoms occupying the same and different sub-lattices, respectively. For a pair of atoms of which at least one is non-magnetic we assume an integral of exchange equal zero. We allow a possibility of the non-symmetrical distribution of magnetic atoms in the system by introducing two concentrations for both the sub-lattices $\alpha$ and $\beta$

$$
c_{\varepsilon}=\frac{2 m_{\varepsilon}}{N}, \quad \varepsilon=\alpha, \beta,
$$

where $m_{\varepsilon}$ is a number of magnetic atoms randomly distributed within sub-lattice $\varepsilon\left(m_{\alpha}+m_{\beta}=m\right)$. For a given distribution $\mathcal{R}$ of magnetic atoms, Hamiltonian of the discussed spin system is written in a following form:

$$
\begin{aligned}
\tilde{H}(\mathcal{R}) & =-\frac{1}{2} \sum_{(r, s)} I_{\varepsilon}(r, s)\left[S_{r}^{z} S_{s}^{z}+\eta_{\varepsilon}\left(S_{r}^{+} S_{s}^{-}+S_{r}^{-} S_{s}^{+}\right)\right] \\
+ & \sum_{(r, t)} K(r, t)\left[S_{r}^{z} S_{t}^{z}+\eta_{\gamma}\left(S_{r}^{+} S_{t}^{-}+S_{r}^{-} S_{t}^{+}\right)\right]-\sum_{(r)} \omega_{0}^{\varepsilon} S_{r}^{z},
\end{aligned}
$$

where $\sum_{(. .)}$is summing over all lattice points coupled with magnetic atoms assuming the lattice points $r$ and $s$ belong to the same sub-lattice $\varepsilon=\alpha$ (for $r, s \in \alpha$ ) or $\varepsilon=\beta$ (for $r, s \in \beta$ ), whereas the lattice points $r$ and $t$ belong to different sub-lattices. Independently of the distribution $\mathcal{R}$, the constants $I_{\varepsilon}(r, s)$ and $K(r, t)$ denote positive integrals of exchange bonding ferromagnetically pairs of magnetic atoms inside each sub-lattice $\varepsilon$ and bonding antiferromagnetically pairs of atoms coupled with different sub-lattices, respectively. Parameters $\eta_{\varepsilon}, \eta_{\gamma} \in\langle 0,1 / 2\rangle$ determine the exchange anisotropy of the relative couplings but the last term of Hamiltonian (2) describes the energy of spin interactions with the external magnetic field $H$, at the same time $\omega_{0}^{\varepsilon}=g_{\varepsilon} \mu_{\mathrm{B}} H$, where $g_{\varepsilon}$ is Landé factor which may take different values for magnetic atoms on different sub-lattices, $\mu_{\mathrm{B}}-$ Bohr magneton.

For an arbitrary magnetic point $r$ we introduce the two-time localized Green function defined as $[10,11]$

$$
G_{r}\left(\tau-\tau^{\prime}\right)=\left\langle\left\langle S_{r}^{+}(\tau) \mid S_{r}^{-}\left(\tau^{\prime}\right)\right\rangle\right\rangle=-\mathrm{i} \theta\left(\tau-\tau^{\prime}\right)\left\langle\left[S_{r}^{+}(\tau), S_{r}^{-}\left(\tau^{\prime}\right)\right]_{+}\right\rangle,
$$


where $\langle\ldots\rangle$ is an average over the grand canonical Gibb ensemble and $\theta$ is an ordinary unit step function. In order to find the Green function one should solve the equation of motion, which in the spectral representation $\omega$ takes the following form:

$$
\omega G_{r}(\omega) \equiv \omega\left\langle\left\langle S_{r}^{+} \mid S_{r}^{-}\right\rangle\right\rangle_{\omega}=\left\langle\left[S_{r}^{+}, S_{r}^{-}\right]_{+}\right\rangle+\left\langle\left\langle\left[S_{r}^{+}, \tilde{H}\right]_{-} \mid S_{r}^{-}\right\rangle\right\rangle_{\omega},
$$

where

$$
\begin{aligned}
& G_{r}\left(\tau-\tau^{\prime}\right)=\frac{1}{2 \pi} \int_{-\infty}^{+\infty} G_{r}(\omega) \exp \left(-\mathrm{i} \omega\left(\tau-\tau^{\prime}\right)\right) \mathrm{d} \omega \\
& G_{r}(\omega)=\int_{-\infty}^{+\infty} G_{r}(\tau) \exp (\mathrm{i} \omega \tau) \mathrm{d} \tau .
\end{aligned}
$$

Using the Tyablikov decoupling scheme [11] in Eq. (4) and neglecting correlations of spin operators located at different lattice points we obtain a linear equation for the Green function $G_{r}(\omega)$ which relates to the molecular field approximation. This approximation neglecting fluctuations of averages of spin operators coupled with different lattice points is the lowest one in the self-consisting procedure of chain system of equations for the Green function. To obtain the next approximation, considering square fluctuations of temperature averages, for the second-order Green function which is in a form of the second term in Eq. (4), the following equation of motion should be written:

$$
\begin{aligned}
& \omega\left\langle\left\langle\left[S_{r}^{+}, \tilde{H}\right]_{-} \mid S_{r}^{-}\right\rangle\right\rangle_{\omega}= \\
& \left\langle\left[\left[S_{r}^{+}, \tilde{H}\right]_{-}, S_{r}^{-}\right]_{+}\right\rangle+\left\langle\left\langle\left[\left[S_{r}^{+}, \tilde{H}\right]_{-}, \tilde{H}\right]_{-} \mid S_{r}^{-}\right\rangle\right\rangle_{\omega} .
\end{aligned}
$$

For the third-order Green function obtained on the right side of the above equation the relevant fourth-order equation can be written and so on. The procedure leads to an infinite chain system of equations that does not have an analytical solution. To self-consist the system of equations, the Green function of the highest order should be decoupled at the given stage of the chain and it should be expressed by the Green functions of lower orders. Irrespectively of the method of decoupling the obtained Green functions usually depend on the localized variables of the following type:

$$
X_{\varepsilon n}(r)=\sum_{(j \neq r)} \Lambda_{\varepsilon n}(r, j)\left\langle\lambda_{\varepsilon n}(j)\right\rangle, \quad n=1,2, \ldots, w,
$$

where $\Lambda_{\varepsilon n}(r, j)$ are given functions of the exchange constants $I_{\varepsilon}(r, j)$ or $K(r, j)$ for pairs of magnetic atoms $(r, j)$ coupled with the same or different sub-lattices whereas $\left\langle\lambda_{\varepsilon n}(j)\right\rangle$ are averages of functions of localized spin operators, usually terms $\left\langle S_{j}^{z}\right\rangle$. The forms of functions $\Lambda$ and $\lambda$ and their number $w$ depend on the method of decoupling of the chain of equations for the Green function as well as on the order 
of equation at which the decoupling is realized. For the geometrically equivalent sub-lattices the number $w$ of functions $\Lambda$ or $\lambda$ is the same for each sub-lattice.

\section{Green function averaging procedure}

Fourier transform $\Lambda_{\varepsilon n}(\boldsymbol{k})$ in the vector space $\boldsymbol{k}$ is assigned to every function $\Lambda_{\varepsilon n}(r, j)$ accordingly to the following relation:

$$
\Lambda_{\varepsilon n}(r, j)=\frac{2}{N} \sum_{\boldsymbol{k}} \Lambda_{\varepsilon n}(\boldsymbol{k}) \exp \left(-\mathrm{i} \boldsymbol{k}\left(\boldsymbol{R}_{r}-\boldsymbol{R}_{j}\right)\right),
$$

where the summing extends over all $N / 2$ reciprocal lattice vectors $\boldsymbol{k}$ within the first Brillouin zone of a sub-lattice. In the site approach the assumption of strictly reverse transformation to transformation (9) is logical [12]

$$
\Lambda_{\varepsilon n}(\boldsymbol{k})=\sum_{r, j} \Lambda_{\varepsilon n}(r, j) \exp \left(\mathrm{i} \boldsymbol{k}\left(\boldsymbol{R}_{r}-\boldsymbol{R}_{j}\right)\right),
$$

where $\Lambda_{\varepsilon n}(\boldsymbol{k})$ and $\Lambda_{\varepsilon n}(r, j)$ have an identical meaning as in Eq. (9).

For every average $\left\langle\lambda_{\varepsilon n}(j)\right\rangle$ we introduce its distribution function in the vector space $\boldsymbol{k}$

$$
\sigma_{\varepsilon n}(\boldsymbol{k})=\sum_{(j)}^{(\varepsilon)}\left\langle\lambda_{\varepsilon n}(j)\right\rangle \exp \left(\mathrm{i} \boldsymbol{k} \boldsymbol{R}_{j}\right)
$$

and structural fluctuation relating to the quantity

$$
\tilde{\sigma}_{\varepsilon n}(\boldsymbol{k})=\sigma_{\varepsilon n}(\boldsymbol{k})-\left\langle\left\langle\sigma_{\varepsilon n}(\boldsymbol{k})\right\rangle\right\rangle .
$$

The index $\varepsilon$ at the sum sign in Eq. (11) states for summing over the points of sublattice $\varepsilon$ and $\langle\langle\ldots\rangle\rangle$ is an average over all possible configurations $\mathcal{R}$ of magnetic atoms in the system and it is defined as:

$$
\begin{aligned}
& \left\langle\left\langle\sigma_{\varepsilon n}(\boldsymbol{k})\right\rangle\right\rangle=\lambda_{\varepsilon n}\left\langle\left\langle\rho_{\varepsilon}(\boldsymbol{k})\right\rangle\right\rangle, \\
& \rho_{\varepsilon}(\boldsymbol{k})=\sum_{(j)}^{(\varepsilon)} \exp \left(\mathrm{i} \boldsymbol{k} \boldsymbol{R}_{j}\right) .
\end{aligned}
$$

The parameters $\lambda_{\varepsilon n}$ do not depend on the lattice point $j$ and they have a meaning of the expectation values of the functions $\left\langle\lambda_{\varepsilon n}(j)\right\rangle$ fluctuating from a lattice point to point

$$
\lambda_{\varepsilon n} \equiv\left\langle\left\langle\lambda_{\varepsilon n}(j)\right\rangle\right\rangle, \quad j \in \varepsilon .
$$

The structural average $\left\langle\left\langle\rho_{\varepsilon}(\boldsymbol{k})\right\rangle\right\rangle$ in Eq. (13) connects to none of the physical parameters and depends only on crystallographic structure of the system as well as on the concentration of magnetic atoms $c_{\varepsilon}$. The following quantity is a measure of the fluctuation of the above parameter

$$
\tilde{\rho}_{\varepsilon}(\boldsymbol{k})=\rho_{\varepsilon}(\boldsymbol{k})-\left\langle\left\langle\rho_{\varepsilon}(\boldsymbol{k})\right\rangle\right\rangle,
$$

where

$$
\left\langle\left\langle\rho_{\varepsilon}(\boldsymbol{k})\right\rangle\right\rangle=\frac{N}{2} c \delta(\boldsymbol{k}), \quad \delta(\boldsymbol{k})=\sum_{j}^{(\varepsilon)} \exp \left(\mathrm{i} \boldsymbol{k} \boldsymbol{R}_{j}\right) .
$$

The summing on the right side of the equation runs for all magnetic as well as 
non-magnetic points of sub-lattice $\varepsilon$. Using the relations of (9-17) and limiting to exchange interactions between the nearest neighbouring atoms we may write expression (8) in a following form:

$$
\begin{aligned}
& X_{\varepsilon l}(r)=\lambda_{\varepsilon l} \Lambda_{\varepsilon l} c_{\varepsilon} z_{l}\left[1+x_{\varepsilon l}(r)\right], \\
& x_{\varepsilon l}(r)=\frac{2}{N} \sum_{\boldsymbol{k}} \frac{\gamma_{l}(\boldsymbol{k})}{c_{\varepsilon}} \exp \left(-\mathrm{i} \boldsymbol{k} \boldsymbol{R}_{r}\right) \tilde{\rho}_{\varepsilon}(\boldsymbol{k}), \\
& \gamma_{l}(\boldsymbol{k})=\frac{1}{z_{l}} \sum_{\delta_{l}} \exp \left(\mathrm{i} \boldsymbol{k} \boldsymbol{\delta}_{l}\right), \quad l=1,2,
\end{aligned}
$$

where for $r \in \alpha(\beta), \varepsilon=\alpha(\beta)$ for $l=1$ or $\varepsilon=\beta(\alpha)$, for $l=2$, but $\boldsymbol{\delta}_{l}$ is a vector determining the locations of $z_{1}$ intra-sub-lattice and $z_{2}$ inter-sub-lattice nearest neighbouring atoms. Parameters $\Lambda_{\varepsilon l}$ refer to the exchange constants $I_{\varepsilon}$ or $K$ but terms $\gamma_{l}(\boldsymbol{k})$ define a spatial structure of the crystal lattice. With regard to the nearest neighbouring atoms any of the Green functions $Q$ then does not depend on fluctuations of many parameters (8) but only on two fluctuating around zero variables $x_{\varepsilon l}$ connecting to the configuration of magnetic atoms on both the sub-lattices

$$
Q_{r}=Q_{r}\left[\omega, x_{\varepsilon 1}(r), x_{\varepsilon^{\prime 2}}(r)\right], \quad \varepsilon \neq \varepsilon^{\prime} .
$$

To estimate the impact of structural fluctuation $\tilde{\rho}_{\varepsilon}(\boldsymbol{k})$ on the Green functions, it is expanded into Taylor's series in relation to variables $x_{\varepsilon l}$

$$
Q_{r}(\omega)=\left[D_{r}^{(2)} Q_{r}\right]_{0}\left(\frac{2}{N}\right)^{\nu} \sum_{\{\boldsymbol{K}\}} \prod_{l, f} \frac{\gamma_{l}\left(\boldsymbol{k}_{l f}\right)}{c_{\varepsilon}} \exp \left(-\mathrm{i} \boldsymbol{k}_{l f} \boldsymbol{R}_{r}\right) \tilde{\rho}_{\varepsilon}\left(\boldsymbol{k}_{l f}\right),
$$

where

$$
\begin{aligned}
& {\left[D_{r}^{(2)} Q_{r}\right]_{0}=\sum_{\nu=0}^{\infty} \frac{1}{\nu !} \sum_{\nu_{1}+\nu_{2}=\nu} \frac{\nu !}{\nu_{1} ! \nu_{2} !}\left[\frac{\partial^{\nu} Q_{r}\left(\omega, x_{\varepsilon 1}, v_{\varepsilon^{\prime} 2}\right)}{\partial x_{\varepsilon 1}^{\nu_{1}} \partial x_{\varepsilon^{\prime} 2}^{\nu_{2}}}\right]_{0},} \\
& \{\boldsymbol{K}\}=\left\{\boldsymbol{k}_{11}, \boldsymbol{k}_{12}, \ldots, \boldsymbol{k}_{1 \nu_{1}}, \boldsymbol{k}_{21}, \boldsymbol{k}_{22}, \ldots, \boldsymbol{k}_{2 \nu_{2}}\right\}, \\
& \prod_{l, f}(\ldots)=\prod_{l=1}^{2} \prod_{f=1}^{\nu_{1}}(\ldots) .
\end{aligned}
$$

The second sum in Eq. (23) denotes summing over all possible combinations $\nu_{1}$ and $\nu_{2}$ so that $\nu_{1}+\nu_{2}=\nu$. transform

In the representation of vectors $\boldsymbol{k}$ the Green function $Q_{r}(\omega)$ relates to the

$$
Q_{\varepsilon}(\boldsymbol{k}, \omega)=\sum_{(r)}^{(\varepsilon)} Q_{r}(\omega) \exp \left(\mathrm{i} \boldsymbol{k} \boldsymbol{R}_{r}\right)
$$

and its averaged over all distributions $\mathcal{R}$ value

$$
\left\langle\left\langle Q_{\varepsilon}(\boldsymbol{k}, \omega)\right\rangle\right\rangle=\frac{N}{2} c_{\varepsilon} Q_{\varepsilon}(\omega) \delta(\boldsymbol{k}) .
$$

The function $Q_{\varepsilon}(\omega)$ does not depend on distribution of magnetic atoms and it may be used to describe the statistical properties of the system. Plugging Eq. (22) into 
Eq. (26) and using the basic rules of the Cumulant Expansion Technique [13] we find

$$
\begin{gathered}
Q_{\varepsilon}(\omega) \delta(\boldsymbol{k})=\left[D_{r}^{(2)} Q_{r}\right]_{0}\left(\frac{2}{N}\right)^{\nu} \sum_{\left\{\boldsymbol{K}_{\}}\right.} \delta\left(\boldsymbol{k}-\sum_{l, f} \boldsymbol{k}_{l f}\right) \\
\quad \times \prod_{l=1}^{2}\left\langle\left\langle\prod_{f=1}^{\nu_{l}} \tilde{\rho}_{\varepsilon}\left(\boldsymbol{k}_{l f}\right)\right\rangle\right\rangle \prod_{f=1}^{\nu_{l}} \frac{\gamma_{l}\left(\boldsymbol{k}_{l f}\right)}{c_{\varepsilon}} .
\end{gathered}
$$

\section{The approximation of high and low structural fluctuations}

The averaged Green function $Q_{\varepsilon}(\omega)$ was presented in a form which allows one to calculate it by summing the terms of the series (28) from all correlations of structural fluctuations connected with a random distribution of magnetic atoms in the system. In the general case the analytical form of the averaged Green function is impossible to calculate so summing the terms of the series (28) is usually realized in approximation. The approximation depends on concentration $c_{\varepsilon}$ of magnetic atoms and is connected with the assessment of the structural fluctuations impact on the Green function and as the consequence on physical properties of the system. Due to its complexity, the problem will be analysed in a separate paper. In the literature authors mostly use two approximations taking into account structural fluctuations in coupling of the lattice points with magnetic atoms. One of them, considered as reliable for high concentrations of magnetic atoms, takes the following form $[14,15]$ :

$$
\left\langle\left\langle\prod_{f=1}^{\nu_{l}} \tilde{\rho}_{\varepsilon}\left(\boldsymbol{k}_{l f}\right)\right\rangle\right\rangle \approx \begin{cases}\prod_{f=1}^{\nu_{l} / 2}\left\langle\left\langle\tilde{\rho}_{\varepsilon}\left(\boldsymbol{k}_{l, 2 f-1}\right)\left\langle\tilde{\rho}_{\varepsilon}\left(\boldsymbol{k}_{l, 2 f}\right)\right\rangle\right\rangle\right. & \text { for even } \nu_{l}, \\ 0 & \text { for uneven } \nu_{l} .\end{cases}
$$

This approximation is strictly satisfied within the limits of high concentrations of magnetic atoms for which structural fluctuations of the lowest orders are more probable than it is for high structural fluctuations. Within the limits of low concentrations another approximation seems more logical $[13,16]$

$$
\left\langle\left\langle\prod_{f=1}^{\nu_{l}} \tilde{\rho}_{\varepsilon}\left(\boldsymbol{k}_{l f}\right)\right\rangle\right\rangle \approx\left\langle\left\langle\prod_{f=1}^{\nu_{l}} \rho_{\varepsilon}\left(\boldsymbol{k}_{l f}\right)\right\rangle\right\rangle_{c}
$$

This approximation when used in the Cumulant Expansion Technique gives good results for the linear range of concentration $c_{\varepsilon}$. The results obtained in numerical analysis for cubic models of Heisenberg ferro- and antiferromagnet in reference to critical concentrations do not distinguish in both the approximations of more than $3 \%$ in their quantity. Such close results are explained by the fact that the greatest contribution to the physical properties comes from the lowest square structural fluctuations which are the same in both the approximations whereas considering all contributions coming from all structural fluctuation of the higher orders leads in both the approximations only to correction of one order lower quantity [17]. 
Both of the approximations give then quite close results. Approximation (29) has an important advantage - when considered in Eq. (28) allows for summing the series and gives the averaged Green function in its analytical form

$$
\begin{gathered}
Q_{\varepsilon}(\omega)=\left\{D_{r}^{(2)} Q_{r}\left[\omega, x_{\varepsilon 1}(r), x_{\varepsilon^{\prime} 2}(r)\right]\right\}_{0} \delta_{\varepsilon 1}^{\nu_{1}}\left(c_{\varepsilon}\right) \delta_{\varepsilon^{\prime} 2}^{\nu_{2}}\left(c_{\varepsilon^{\prime}}\right) \\
=\frac{1}{4} \sum_{i, j= \pm 1} Q_{r}\left[\omega, i \delta_{\varepsilon 1}\left(c_{\varepsilon}\right), j \delta_{\varepsilon^{\prime} 2}\left(c_{\varepsilon^{\prime}}\right)\right]
\end{gathered}
$$

where

$$
\delta_{\varepsilon l}\left(c_{\varepsilon}\right)=\left[\frac{c_{\varepsilon}-c_{\varepsilon}^{2}}{c_{\varepsilon}^{2}} \frac{2}{N} \sum_{\boldsymbol{k}} \gamma_{l}^{2}(\boldsymbol{k})\right]^{\frac{1}{2}}, \quad l=1,2, \quad \varepsilon=\alpha, \beta .
$$

The analytical form of Green function allows one to calculate basic physical parameters of a system such as magnetization and internal energy. Due to it the full analysis of magnetic and heat processes occurring in a system under influence of temperature changes and concentrations of the magnetic components as well as external magnetic field for different crystallographic structures and for different lattice and exchange anisotropy are possible.

\section{Magnetization in the external magnetic field}

To illustrate the magnetization processes occurring in a two-sub-lattice dilute metamagnet the authors used the approximation of a given effective magnetic field that neglects the correlations of the spin operators located at different lattice sites. To go beyond the molecular field approximation, the self-consisting procedure of the chain equations system for the Green function at its second stage is performed. It is carried out by means of the modified Tyablikov decoupling scheme, introduced by Riess and Movroyannis for pure magnetic systems [18, 19]

$$
\left\langle\left\langle S_{s}^{z} S_{s^{\prime}}^{z} S_{r}^{+} \mid S_{r}^{-}\right\rangle\right\rangle_{\omega} \approx\left\langle S_{s}^{z} S_{s^{\prime}}^{z}\right\rangle G_{r}(\omega), \quad s, s^{\prime} \neq r .
$$

Using assumption (33) in Eqs. (4) and (7) and taking into account approximation (29) allow one to determine the analytical form of the Green function averaged over all possible configurations of magnetic atoms in the system.

$$
G_{\varepsilon}(\omega)=\frac{1}{4} \sum_{i, j= \pm 1} \frac{\omega-\omega_{0}^{\varepsilon}+\Gamma_{i j}^{\varepsilon}}{\left(\omega-\omega_{0}^{\varepsilon}+\Delta_{i j}^{\varepsilon}\right)\left(\omega-\omega_{0}^{\varepsilon}-\Delta_{i j}^{\varepsilon}\right)},
$$

where

$$
\begin{aligned}
\Delta_{i j}^{\varepsilon} & =\left[\left(\Gamma_{i j}^{\varepsilon}\right)^{2}+\left(\Omega_{i j}^{\varepsilon}\right)^{2}\right]^{1 / 2}, \\
\Gamma_{i j}^{\varepsilon} & =I_{\varepsilon}\left\langle S_{\varepsilon}^{z}\right\rangle z_{1} c_{\varepsilon}\left(1+i \delta_{\varepsilon 1}\right)-K\left\langle S_{\varepsilon^{\prime}}^{z}\right\rangle z_{2} c_{\varepsilon^{\prime}}\left(1+j \delta_{\varepsilon^{\prime} 2}\right), \varepsilon=\alpha, \beta, \varepsilon \neq \varepsilon^{\prime}, \\
\Omega_{i j}^{\varepsilon} & =\left[I_{\varepsilon}^{2}\left(\frac{1}{4}+\eta_{\varepsilon}^{2}-\left\langle S_{\varepsilon}^{z}\right\rangle^{2}\right) z_{1} c_{\varepsilon}\left(1+i \delta_{\varepsilon 1}\right)\right. \\
& \left.+K^{2}\left(\frac{1}{4}+\eta_{\gamma}^{2}-\left\langle S_{\varepsilon^{\prime}}^{z}\right\rangle^{2}\right) z_{2} c_{\varepsilon^{\prime}}\left(1+j \delta_{\varepsilon^{\prime} 2}\right)\right]^{1 / 2} .
\end{aligned}
$$


The averaged Green function, with the known spectral relations, determines the average moment $\left\langle S_{\varepsilon}^{z}\right\rangle$ connected with any magnetic lattice site on each sub-lattice $\varepsilon$

$$
\begin{aligned}
\left\langle S_{\varepsilon}^{z}\right\rangle & =\frac{\mathrm{i}}{4 \pi} \lim _{o \rightarrow 0} \int_{-\infty}^{+\infty}\left[G_{\varepsilon}(\omega+\mathrm{io})-G_{\varepsilon}(\omega-\mathrm{i} o)\right] \tanh \left(\frac{1}{2} \beta \omega\right) \mathrm{d} \omega \\
& =\frac{1}{16} \sum_{i, j= \pm 1}\left\{\left(1-\frac{\Gamma_{i j}^{\varepsilon}}{\Delta_{i j}^{\varepsilon}}\right) \tanh \left[\frac{1}{2} \beta\left(\omega_{0}^{\varepsilon}-\Delta_{i j}^{\varepsilon}\right)\right]\right. \\
& \left.+\left(1+\frac{\Gamma_{i j}^{\varepsilon}}{\Delta_{i j}^{\varepsilon}}\right) \tanh \left[\frac{1}{2} \beta\left(\omega_{0}^{\varepsilon}+\Delta_{i j}^{\varepsilon}\right)\right]\right\} .
\end{aligned}
$$

The basic equations (38) fully determine magnetization $M$ of a system and the difference of sub-lattice magnetizations $m$ as a function of temperature $T$, concentration $c_{\varepsilon}$, and the external magnetic field $H$ :

$$
\begin{aligned}
M & =\frac{1}{2} N \mu_{\mathrm{B}}\left[g_{\alpha}(S+s)+g_{\beta}(S-s)\right], \\
m & =\frac{1}{2} N \mu_{\mathrm{B}}\left[g_{\alpha}(S+s)-g_{\beta}(S-s)\right],
\end{aligned}
$$

where $S$ and $s$ are the parameters of the long-range order

$$
\begin{aligned}
& S=\frac{1}{2}\left(\sigma_{\alpha}+\sigma_{\beta}\right), \\
& s=\frac{1}{2}\left(\sigma_{\alpha}-\sigma_{\beta}\right)
\end{aligned}
$$

but

$$
\sigma_{\varepsilon}=c_{\varepsilon}\left\langle S_{\varepsilon}^{z}\right\rangle
$$

is an average magnetic moment relating to every magnetic as well as non-magnetic lattice point of the sub-lattice $\varepsilon$.

For $c_{\alpha}=c_{\beta}$ and $H \neq 0$, within the whole range of temperatures, the parameter $S$ and magnetization $M$ are always different from zero. From the analysis of Eqs. (38) it results that for the temperatures higher than Néel's critical temperature $T_{\mathrm{N}}$, i.e. for the temperatures at which the system for $H \rightarrow 0$ was in a paramagnetic state, every value of the field $H$ corresponds with only one solution $\left\langle S_{\varepsilon}^{z}\right\rangle=\left\langle S_{\varepsilon^{\prime}}^{z}\right\rangle$. It means that the parameter $s$ and the difference of sub-lattice magnetizations $m$ always equal zero and the system is in the paramagnetic state, due to the ferromagnetic spin configuration sometimes called induced ferromagnetism. For the temperatures $T<T_{\mathrm{N}}$, but higher than a given temperature $T_{\text {trc }}$, every value of the field $H$ relates to the unique solution $s \neq 0$ or $s=0$. Figure 1 shows, for this case, the typical dependence of the long-range order parameters and the relative magnetization of both the sub-lattices as a function of the external magnetic field. When there are unique solutions for $S$ and $s$, the second derivative of the free energy 


$$
F(H)=F\left(H_{0}\right)-\int_{H_{0}}^{H} M\left(H^{\prime}\right) \mathrm{d} H^{\prime}
$$

in relation to the field at the point separating the states with the solutions $s \neq 0$ and $s=0$ is discontinuous. The phase transition induced by the external magnetic field is then the second-order transition. From the basic equations analysis it results that at a given concentration of magnetic atoms, the lower temperature relates to the higher value of the critical field. In the limit of $K \rightarrow 0$, i.e. for the dilute ferromagnet the phase transition induced by the external magnetic field vanishes. The critical concentration $c_{0}$ is the low limit of the magnetic atoms concentration at which any phase transitions in a system are possible.
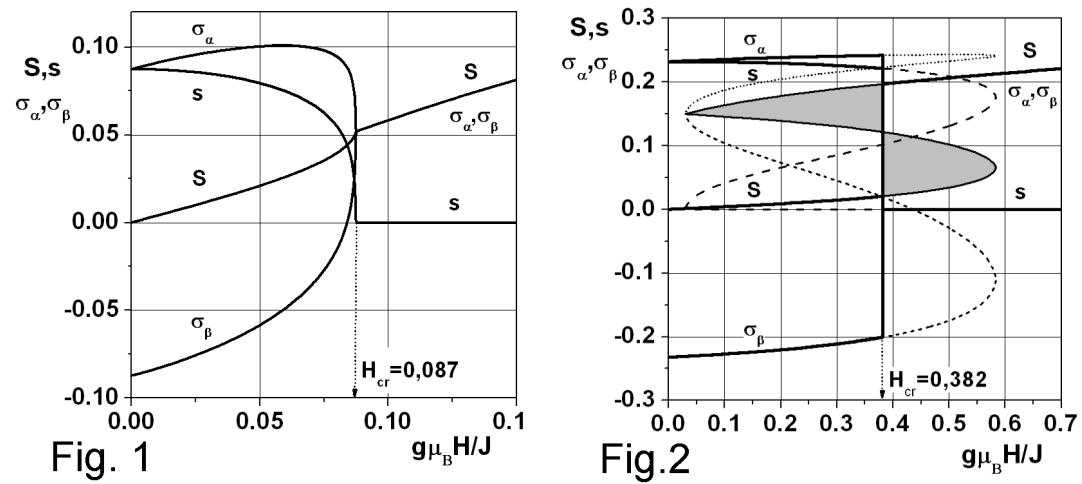

Fig. 1. The long-range order parameters $S$ and $s$ and reduced sub-lattice magnetizations $\sigma_{\varepsilon}$ as a function of the external magnetic field $H$ at the second-order phase transition $\left(z_{1}=z_{2}=6, K=0.1 J, \eta_{\varepsilon}=\eta_{\gamma}=0.5, c_{\alpha}=c_{\beta}=0.6, k T_{\mathrm{N}} / J=0.794\right.$, $\left.T=0.95 T_{\mathrm{N}}\right)$.

Fig. 2. The long-range order parameters $S$ and $s$ and reduced sub-lattice magnetizations $\sigma_{\varepsilon}$ as a function of the external magnetic field $H$ at the first-order phase transition. The bold solid lines determine the changes in the parameters $S, s, \sigma_{\varepsilon}$ corresponding to the minimum free energy $\left(z_{1}=z_{2}=6, K=0.1 J, \eta_{\varepsilon}=\eta_{\gamma}=0.5, c_{\alpha}=c_{\beta}=0.6\right.$, $\left.k T_{\mathrm{N}} / J=0.794, T=0.5 T_{\mathrm{N}}\right)$.

At the sufficiently low temperatures $T<T_{\text {trc }}$, functions $S(H)$ and $s(H)$, derived from the basic equations, are not always unique functions of the field $H$. For the given range of values $H$, the system of Eqs. (38) has even three pairs of solutions for $S$ and $s$ (Fig. 2). To choose the physically appropriate field dependence of the parameters $S$ and $s$, the solution, whose the minimum free energy relates to, should be selected from all the solutions. The other solutions that relate to non-physical or metastable states are discarded. From Fig. 3 showing the dependence of the free energy $F$ on the field $H$ we can see that the minimum free energy corresponds to the solutions: $s \neq 0$ for $H<H_{\mathrm{cr}}$ (antiferromagnetic order) and $s=0$ for $H>H_{\mathrm{cr}}$ (phase of induced ferromagnetism). The derivative of the 

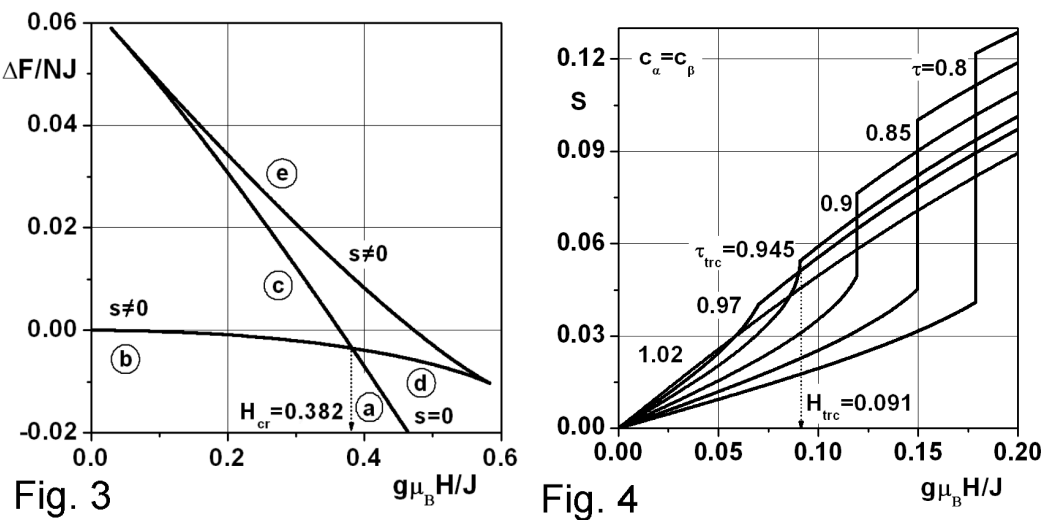

Fig. 3. The free energy change $\Delta F=F(H)-F(0)$ as a function of the external magnetic field $H$ at the first-order phase transition. Segments (a) and (b) of the free energy relate to the paramagnetic and antiferromagnetic solutions, respectively; (c) and (d) correspond to metastable states but (e) corresponds to the non-physical state $\left(z_{1}=z_{2}=6, K=0.1 J, \eta_{\varepsilon}=\eta_{\gamma}=0.5, c_{\alpha}=c_{\beta}=0.6, k T_{\mathrm{N}} / J=0.794, T=0.5 T_{\mathrm{N}}\right)$.

Fig. 4. The long-range order parameter $S$ as a function of the external magnetic field $H$ for various reduced values of temperature $\tau=T / T_{\mathrm{N}}\left(z_{1}=z_{2}=6, K=0.1 J\right.$, $\left.\eta_{\varepsilon}=\eta_{\gamma}=0.5, c_{\alpha}=c_{\beta}=0.6, k T_{\mathrm{N}} / J=0.794\right)$.

free energy in relation to the field is discontinuous at the point $H_{\mathrm{cr}}$ dividing the states of the system with the solutions $s \neq 0$ and $s=0$. It means that the phase transition induced by the external magnetic field is the first-order transition with the abrupt change in magnetization. It is worth noticing that the critical field $H_{\mathrm{cr}}$, reached from the minimum free energy is identical to the critical field obtained by means of the equal area method. The conclusions are presented in Fig. 4 that shows the curves of magnetization of the system as a function of the external magnetic field within the range of the discussed temperatures. The tricritical point $\left(H_{\mathrm{trc}}\right.$, $\left.T_{\text {trc }}\right)$ determining the change in the type of the phase transition occurs only in the magnets with two kinds of exchange interaction and when the concentrations of magnetic atoms on both the sub-lattices are equal. From the numerical analysis it also results that the lower concentration corresponds with lower values of $H_{\text {trc }}$ and $T_{\mathrm{trc}}$. In the limit of the critical concentration any phase transitions in the system vanish and $H_{\mathrm{trc}}, T_{\mathrm{trc}} \rightarrow 0$.

For $c_{\alpha} \neq c_{\beta}$, analogously as for the equal concentrations of magnetic atoms on the sub-lattices, the parameter $\mathrm{S}$ is always the increasing function of the magnetic field $H$. For $H \rightarrow \infty,\left\langle S_{z}\right\rangle \rightarrow 1 / 2$ for every $\varepsilon$ and accordingly to definitions (41), (43) the maximum value of $S$

$$
S_{\infty}=\frac{1}{4}\left(c_{\alpha}+c_{\beta}\right)
$$

depends only on the sum of concentrations of magnetic atoms on both the sub- 
-lattices. It means that at the constant total concentration $c=\left(c_{\alpha}+c_{\beta}\right) / 2$, the saturation magnetization of the system does not depend on the ratio of concentrations of magnetic atoms on the sub-lattices. The behaviour of the parameter $s(H)$ being a measure of the difference between magnetizations of the sub-lattices is a significant component distinguishing the processes of magnetization in a case of equal and different concentrations of magnetic atoms. For $c_{\alpha}=c_{\beta}$ the parameter $s$ is always the decreasing function of $H$, it makes for zero continuously or stepwise at the point of the phase transition and stays equal zero at the further increase in the magnetic field. For $c_{\alpha} \neq c_{\beta}$ and sufficiently high values of the field, the parameter $s$ goes asymptotically towards the constant value

$$
s_{\infty}=\frac{1}{4}\left(c_{\alpha}-c_{\beta}\right)
$$

that depends only on the difference between the concentrations of the magnetic atoms on both the sub-lattices. In the state of induced ferrimagnetism both the parameters $S$ and $s$ are for $T \geq T_{\mathrm{N}}$ always different from zero as distinguished from the case of physically equivalent sub-lattices.

For $T \geq T_{\mathrm{N}}$, at any ratio of the magnetic atoms concentrations on the sub-lattices there is only one solution $S(H)$. In particular, when exchanging $c_{\alpha}$ for $c_{\beta}$ and retaining the total concentration $c=\left(c_{\alpha}+c_{\beta}\right) / 2$, the curve of magnetization is described by the same function $S(H)$. For $T<T_{\mathrm{N}}$ and $c_{\alpha} \neq c_{\beta}$ the basic equations may have one or more solutions for $S$ and $s$. Figure 5 illustrates the dependence of the long-range order parameters on the magnetic field for $c_{\alpha}>c_{\beta}$ and for the temperature at which the system, under the influence of the magnetic field, undergoes the first-order transition. This type of transitions proceeds only within the sufficiently low range of temperatures. For the temperatures out of the
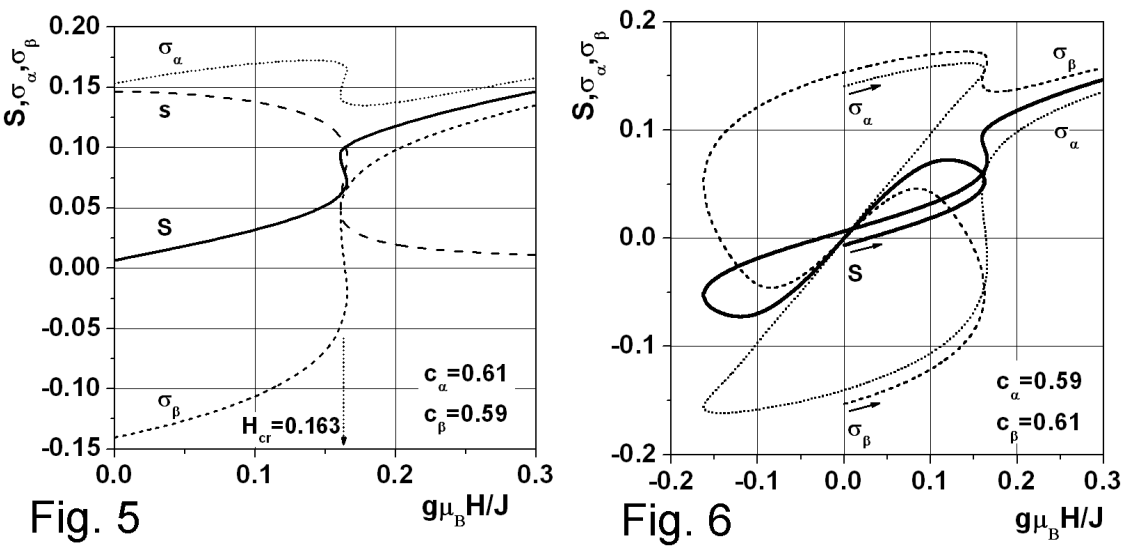

Fig. 5. Graphic presentation of the basic equations solution for $c_{\alpha}>c_{\beta}$ at the first-order phase transition $\left(z_{1}=z_{2}=6, K=0.1 J, \eta_{\varepsilon}=\eta_{\gamma}=0.5, k T_{\mathrm{N}} / J=0.795, T=0.849 T_{\mathrm{N}}\right)$. Fig. 6. Graphic presentation of the basic equations solution for $c_{\alpha}<c_{\beta}$ at the first-order phase transition $\left(z_{1}=z_{2}=6, K=0.1 J, \eta_{\varepsilon}=\eta_{\gamma}=0.5, k T_{\mathrm{N}} / J=0.795, T=0.849 T_{\mathrm{N}}\right)$. 
range, even for $T<T_{\mathrm{N}}$, as distinguished from the case $c_{\alpha}=c_{\beta}$, the first and the second derivatives of the free energy are continuous functions of the field $H$ and the system does not undergo any phase transition in the external magnetic field. Hence, in the phase diagram showing the dependence of the critical field on the temperature for $c_{\alpha} \neq c_{\beta}$, the curve of the phase transitions (the first-order transitions) will end at the critical end-point.

For $T<T_{\mathrm{N}}$ and $c_{\alpha}<c_{\beta}$ the solution of the basic equations has a mathematically different form, comparing it with the case $c_{\alpha}>c_{\beta}$. Within the given range of the field $H$ two different solutions for $S$ and $s$ additionally appear. A formal change in the solutions of the basic equations in particular undergoes while changing concentration $c_{\alpha}$ for $c_{\beta}$. Comparing Figs. 5 and 6 it results that the picture of the solutions significantly changes even at very close concentrations of magnetic atoms on the sub-lattices. However, from Fig. 7 that shows the dependence of the free energy on the magnetic field, it results that in both discussed cases the common segment of the solution corresponds to the states of the system with the same - the lowest values of the free energy. Apparently different solutions come to one equivalent dependence $S(H)$ presented for various temperatures in Fig. 8 . The foregoing result seems logic as the two systems in which the change in the concentrations of the magnetic atoms on the geometrically equivalent sub-lattices was performed, are physically undistinguished and formally different solutions are the result of the asymmetry imposed by the field $H$.
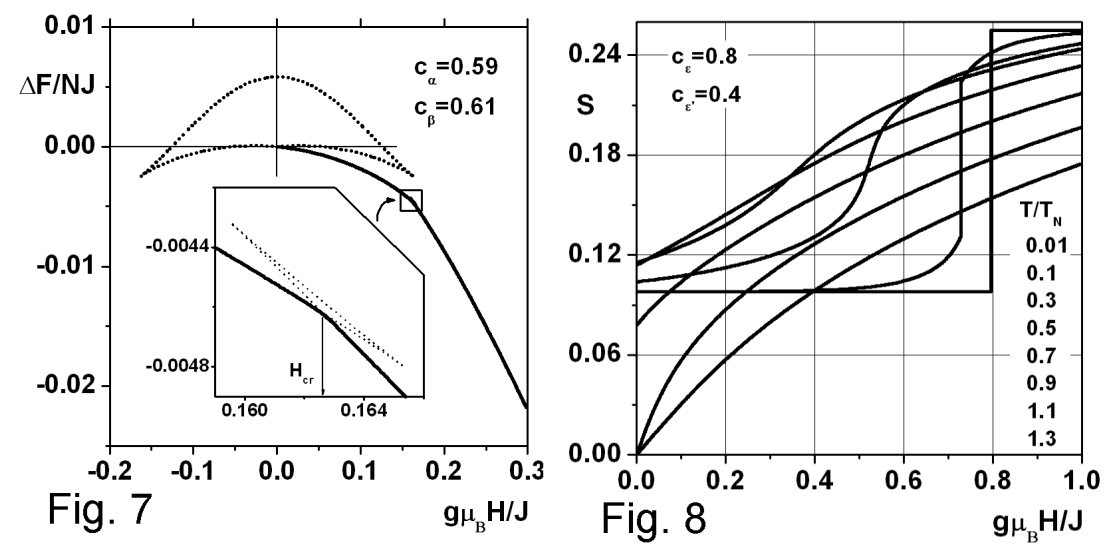

Fig. 7. The free energy change $\Delta F=F(H)-F(0)$ as a function of the external magnetic field $H$ for $c_{\alpha}<c_{\beta}$ at the first-order phase transition. The continuous segment of the diagram corresponds to the lowest free energy states and is the same as for the case $c_{\alpha}=0.61, c_{\beta}=0.59\left(z_{1}=z_{2}=6, K=0.1 J, \eta_{\varepsilon}=\eta_{\gamma}=0.5, k T_{\mathrm{N}} / J=0.795\right.$, $\left.T=0.849 T_{\mathrm{N}}\right)$.

Fig. 8. The long-range order parameter $S$ as a function of the external magnetic field $H$ for various temperatures $T$ at the non-symmetrical distribution of magnetic atoms in a system $\left(z_{1}=z_{2}=6, K=0.1 J, \eta_{\varepsilon}=\eta_{\gamma}=0.5, k T_{\mathrm{N}} / J=1.017\right)$. 


\section{Summary}

There have been analyzed the magnetization processes and the field-induced phase transitions in dilute two sub-lattice metamagnets with mixed ferro- and antiferromagnetic exchange interactions. In a particular model with the symmetrical distribution of magnetic atoms on the sub-lattices, two kinds of phase transitions induced by the external magnetic field occur. The first-order phase transitions undergo in the relatively low range of temperatures and they are separated from the second-order phase transitions by the tricritical point. Both the types of transitions take place below Néel's point only and disappear at the critical concentration as well as in the limit of a dilute magnet with one kind of exchange interactions. For asymmetrical distribution of magnetic atoms within both the sub-lattices the tricritical point vanishes and the only possible phase transitions induced by the external magnetic field are the first-order transitions. These transitions proceed only within the sufficiently low range of temperatures. For the temperatures out of the range, the system does not undergo any phase transition even for temperatures lower than the critical temperature.

It is worth noticing that the results presented for a two-sub-lattice model can also be applied for a dilute system without sub-lattice structure where all the lattice sites may be randomly occupied by magnetic atoms of different kinds and non-magnetic impurities with the same probability. The results obtained for both the models were found to be similar and in the particular case of $z_{1}=z_{2}$ they are even quantitatively very close. For example, the magnetization curves presented for $z_{1}=z_{2}=6$ in Figs. 4, 8 wholly reproduce, within the assumed accuracy, the corresponding results obtained for a dilute system with the coordination number $z=12$ and the same global concentration $c$. For $z_{1} \neq z_{2}$, a transition from a two-sub-lattice model to a dilute system without sub-lattice structure influences much more substantially the magnetization properties of the system. In particular, such a transition decreases the critical temperature and the critical field and makes the system less stable.

\section{References}

[1] Y. Kasai, S. Miyazima, I. Syozi, Prog. Theor. Phys. 42, 1 (1969).

[2] F. Matsubara, Prog. Theor. Phys. 51, 1694 (1974).

[3] F. Matsubara, Prog. Theor. Phys. 53, 1603 (1975).

[4] Y. Ueno, T. Oguchi, Prog. Theor. Phys. 54, 642 (1975).

[5] Y. Itoh, K. Teramoto, Prog. Theor. Phys. 57, 28 (1977).

[6] T.A.L. Ziman, R.J. Elliott, J. Phys. C, Solid State Phys. 13, 845 (1980).

[7] M. Błaszyk, B. Fechner, Acta Phys. Pol. A 97, 855 (2000).

[8] N. Shannon, B. Schmidt, K. Penc, P. Thalmeier, Eur. Phys. J. B 38, 599 (2004).

[9] R.S. Wadas, Magnetism in Spinels, Garnets and Perovskites, PWN, Warszawa 1974. 
[10] D.N. Zubariew, Termodynamika statystyczna, PWN, Warszawa 1974.

[11] S.W. Tyablikov, Metody kwantowoy teorii magnetizma, NAUKA, Moskva 1975.

[12] A.B. Harris, P.L. Leath, B.G. Nickiel, R.J. Elliott, J. Phys. C 7, 1693 (1974).

[13] F. Yonezawa, T. Matsubara, Prog. Theor. Phys. 35, 357,759 (1966).

[14] T. Kaneyoshi, R. Honmura, J. Phys. C 5, L65 (1972).

[15] T. Kaneyoshi, J. Phys. C 5, L107 (1972).

[16] K. Yamamoto, T. Koneyoshi, Prog. Theor. Phys. 50, 1416 (1973).

[17] B. Fechner, R. Pikuła, Phys. Lett. A 82, 429 (1981).

[18] I. Riess, Physica 74, 496 (1974).

[19] I. Riess, C. Movroyannis, Physica 75, 483 (1974). 